\title{
Research on Key Problems of Making 3D Scene Model Based on VRML Yang Kuang ${ }^{1, a}$, Jie Jiang ${ }^{2, b}$ \\ ${ }^{1}$ Institute of Education, Jiang Xi Science and Technology Normal University, NanChang, China \\ ${ }^{2}$ Institute of Education, Jiang Xi Science and Technology Normal University, NanChang, China \\ aemail: ky_super@163.com, bemail: janyjiang@163.com
}

\section{Keywords: Virtual Reality; VRML; Model; Bake; Lod}

\begin{abstract}
In recent years, with the development of computer technology, virtual reality technology has been paid more and more attention. The scene construction is the foundation of the realization of VRML virtual reality interactive work. For large-scale virtual scene, the amount of data and the mapping file network transmission will be very large, the interpretation and execution speed have a great impact. This paper focuses on some key problems in virtual reality modeling language. It expected to solve the VRML scene and large amount of data on the network implementation problems of low efficiency, such as how to baked mapping in VRML; render detail level of scene; segmentation method and how to create a virtual character etc.
\end{abstract}

\section{Introduction}

VRML descript 3D scene by using text information, it transmit in Internet, then generate 3D scene in the local machine by VRML browsers. The working mechanism, avoid the transmission of graphics files directly in the network problems, sent the complicated task to the local machine, so as to reduce the burden of network, and make the three dimensional interactive is possible on the Internet [1]. The viewer need a plug-in to observe the VRML scene in the browser. The plug-in is VRML browser, the common plug-in has Blaxxun Contact, CosmoPlayer and so on.

The objects in the VRML scene can be called node. It concluded geometry, material, texture, light, view and so on. People described objects and attributes in the scenario with node. The node constitute virtual scene according to the certain rule. Node in the scene graph are used to demonstrate the virtual objects from the sense of vision and hearing, they follow the hierarchy are combined together, the performance of the scene space effect. The following will discuss key issues of building scene.

\section{Mapping and Baking}

Shadow concept does not normally support in VRML, produce the lighting effects in the VRML scene going through the baking technology, it will generate light to 3D object map. Render To Textures is method of generate the illumination information in 3D software to a mapping mode, and then put the baked mapping back up the objects in the scene technology. Through the software mapping, it can add on three-dimensional objects. When the 3D model map is baked, added to the VRML scene. As the mapping produced light, so the 3D object in the VRML scene do not need the renderer support can have light effect, produce the real situation.

Compare shadows of baked objects in VRML, as shown in figure. For the VRML scene modeling, texture baking can enhance the actual effect in the scene, texture baking is a method in VRML, it is the fastest speed to get the best performance of the 3D effect.
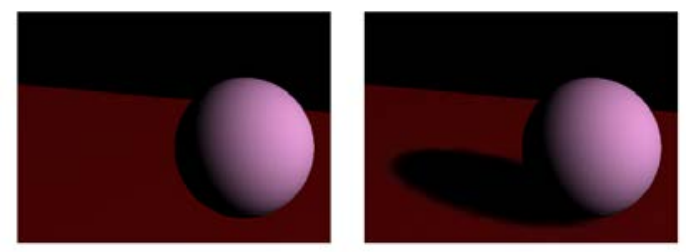

Fig.1. Shadow object after roasting in VRML scene 


\section{Level of Detail}

LOD can improve the speed of VRML scene loading. Objects can be rendered image size changes or level of detail based on geometric complexity. In the real world, people see the same object from different distance, this object is different in the level of detail. Also in the virtual scene, when the objects are relatively far away from the point of view, people only to see the outlines of the object. For the same object can be produced by several different details effect model. The final result is, the viewpoint is far from the object, the selection model display rough details, when the distance is near, it display more details of model.

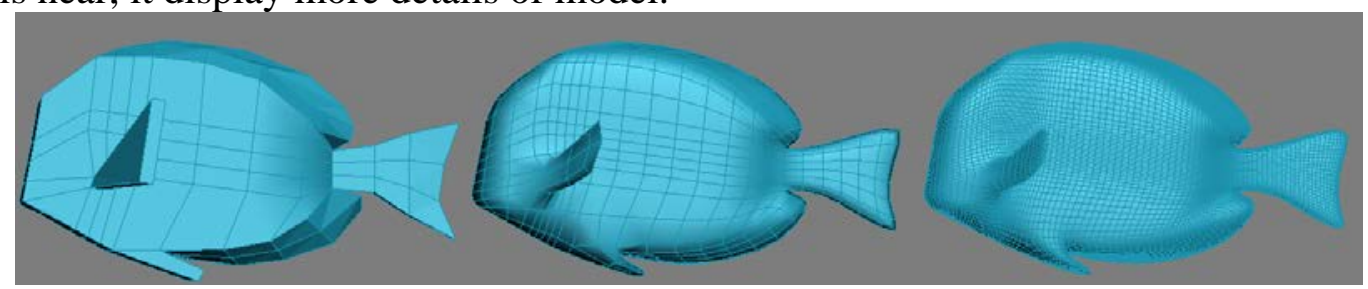

Fig.2. Three different details of the object model

Using the level of detail in rendering, view from the model 100 units within the right in the photo above shows the model, view from the model of distance is between 100 to 500 units, the image shows the intermediate models, it is more than 500 units of distance from the model view shows the left most above model, code is expressed as follows:

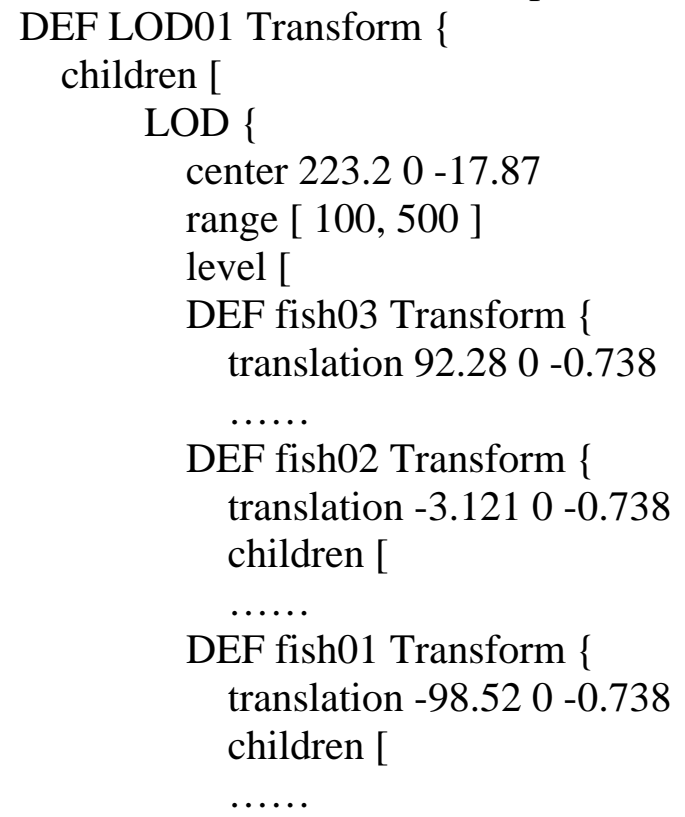

\section{Segment Scene}

There are a large number of models in complex 3D scene. Use a single VRML file to realize all the virtual scene waste system resources, and debugging is not convenient, also cause unknown error. Users open the VRML scene file through the network, it need a complete VRML file. If all scene models are stored in a file, the user watch through the network, model rendering speed will be greatly reduced, so it need segment large scene.

The big scene segmentation is mean the scene is divided into several small modules, they are stored as part of the scene in a new VRML file. Use inline node, in the construction of the whole scene, complete module calls in the need to insert a partial module position using the Inline node address, it can be specified in the material file, module can easily add into the whole scene big scene. The Inline node is described as follows.

Inline

\{

ExposedField MFString url [ ] 
ExposedField SFVec3f bboxCenter 0.0 0.0 0.0

ExposedField SFVec3f bboxSize 5.0 5.0 5.0

\}

Sometimes, there are large number of the same models in the scene, such as street lights and trees. In VRML, you can use the DEF command to define any object in the scene, reuse the USE command in the back of the program, thus save a large amount of system resources. Node definition and reference syntax is defined as follows:

DEF tree2 Transform \{

......

USE tree1

\}

In addition, VRML scene file will become large because of textures. When we finish the scene textures, people need compress it. Generally, people use the JPG or GIF format, which can reduce the occupied size of pictures. The VRML file can be used to compress the VRML file using the gzip tool software, the file will be compressed and only 1/10 of the original size, and it do not affect the execution. In addition, people also can use VrmlPad compression storage or distribution wizard to reduce the size of the VRML file, to achieve the same objective of optimizing scene file.

\section{Sky Box}

Both the production of indoor or outdoor 3D scene, people need skybox to heighten the whole environment, the sky box (Skybox) as the whole scene environment and background. The sky box is composed of six images of the cube, which surrounds the whole scene model. There are two kinds of method make the sky box: one kind is the real environment sky boxes, were filmed in the real environment using fish eye lens; the other is a virtual environment sky boxes, generated by the software, such as in 3DS MAX. In the virtual scene, placing a sphere as a viewpoint, diffuse environment map and set the Reflect/Refract type of the ball, and then the ball can reflect and display the environment as map rendering, can render six images, they are the upper, the lower, the left, the right, the front and the rear, so we can acquire a virtual environment sky box. Below is the environment, people produce it through VUE, and then put into 3DS MAX.

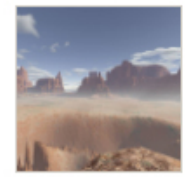

back

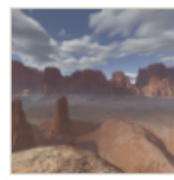

front

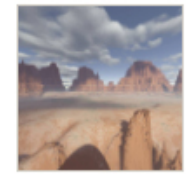

left

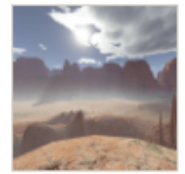

right

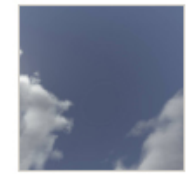

back

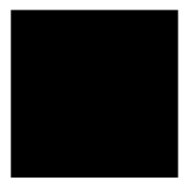

bottom

Fig.3. The different face of the model

\section{Modeling of Virtual Characters}

A method is: making a good character animation in 3DS MAX (including the model and character motion), then make use of the BS Exporter for 3DS MAX, will export multiple texture 3DS MAX role model for baking, skeletal system and other information for the WRL file, but it only supports character studio skeleton skin system are derived, so use this methods need to master 3DS MAX skinned mesh method, the following method is more complex.

Another method is: using Avatar Studio making virtual characters is very convenient, it has very high production efficiency, people can quickly create VRML virtual characters, and can produce a simple interactive action.

\section{Conclusion}

Generally, people making VRML scene use the 3DS MAX and Maya visualization tool for modeling and rendering of rigid body and soft body animation work. The tool converts it to VRML file[3]. The VRML file is large, it is difficult to realize the interaction between users and VRML 
scene. VRML access mode is based on "client / server" mode, in which the server provides the VRML file (suffix WRL) and supporting documents to customers through the network to download resources that you want to access, and through the local platform on the VRML browser access to the Virtual World. Because the browser is provided by a local platform, thus realized the hardware platform is independent [4]. By the VRML working mode shows, making VRML virtual scene need to consider the following factors: the size of the file, the scene files rendering speed, complexity of the scene description. This paper discuss these problems based on the two aspects.

VRML scene model is based of the production of VRML virtual reality, only the scene model is perfect, we can create a VRML virtual reality products which can interact with the user. This paper discussed is the use of visualization software tools and native VRML model combined with the way of making VRML scene model, expected to solve the problems encountered in VRML.

\section{References}

[1] Yanqing YE, Jianlong Shao. Optimization analysis of VRML technique. Journal of Yunnan University of Nationalities: Natural Science Edition [J ] . 2004, 3 (2): 122-124.

[2] Wujun Zhang, VRML virtual reality technology basis and practical tutorial [M]. BeiJing, Metallurgical Industry Press, 2008

[3] Peijun Wang, Research on remote real time 3D Furniture Customization System Oriented Virtual Reality Based on user[J] . Chinese mechanical engineering,2004, 15(12): 1073-1076.

[4] Meng Zhang, Real time scheduling of large-scale virtual scene and loading method based on VRML [J].Software Review,2011,2(2). 